\title{
FUNDAMENTAL EXPERIMENT FOR UTILIZING LIDAR SENSOR FOR RAILWAY
}

\author{
NORIYUKI SHINODA ${ }^{1}$, TOSHIHIRO TAKEUCHI ${ }^{1}$, NOZOMI KUDO ${ }^{1}$, TAKESHI MIZUMA $^{2}$ \\ ${ }^{1}$ National Traffic Safety and Environment Laboratory, National Agency for Automobile and Land Transport \\ Technology, Tokyo \\ ${ }^{2}$ Department of Advanced Energy, Graduate School of Frontier Sciences, University of Tokyo, Chiba
}

\begin{abstract}
At the National Traffic Safety and Environment Laboratory, we proposed a train control system using satellite positioning and general-purpose radio. However, in sections such as tunnels where satellite positioning is not possible, it is necessary to combine this with other methods of determining the absolute position. Therefore, we considered a method to accurately determine the absolute position of the vehicle by ascertaining the feature points by using a Light Detection and Ranging (LiDAR) sensor. In addition, as validation of the performance of the single LiDAR sensor, we installed a LiDAR sensor on a train to study its detection of humans and cars. Through validation of its performance in addition to its potential for absolute position detection, we tried to assess its effectiveness as an obstacle detection device for the future. The probability of accidents between an automobile and a tram is higher at an intersection. Hence, a specific aim of the basic experiments was to ascertain the possibility of using the LiDAR sensor to judge the approach of a car turning right onto a tram track at an intersection. The results of these experiments are reported here.

Keywords: absolute position, LiDAR sensor, obstacle detection, satellite positioning, three-dimensional space recognition sensor.
\end{abstract}

\section{INTRODUCTION}

Local railways are looking to reduce the cost of equipment maintenance in the face of an increasingly difficult economic environment owing to factors such as declining population. We therefore propose a train control system using satellite positioning and general-purpose radio that is aimed at reducing the burden on regional railway operators. However, in view of the presence of sections where satellite positioning is not possible, such as under tunnels or overpasses and also owing to the occurrence of positioning errors owing to multipaths, it is considered necessary to combine this with an understanding of the absolute position by other methods in order to ensure the accuracy of the position information [1].

The ground element used in Automatic Train Stop, the correction of absolute position by other means such as radio frequency identification and position correction during motion by a tachogenerator are some of the methods considered to be effective in accurately determining the absolute position of a vehicle [2].

In addition, with the remarkable advancements in the automatic driving technology of automobiles, the combination of image sensors (measuring the distance of the target by stereo vision), millimeter-wave radar (measuring the distance using the phase difference of the wave reflected by the millimeter wave from the object) and Light Detection and Ranging (LiDAR) sensors (measuring the distance using the time until a projected laser is reflected back from an object) is being extensively used for obstacle detection [3].

We explored the possibility of generalizing these techniques and utilizing them for the detection of the absolute position of vehicles. In this study, we focus on the LiDAR sensor, which is weather resistant and is capable of reasonably accurate detection, from the perspective of application in railways. 
Therefore, we examine the possibility of onboard detection of the absolute position by mounting this LiDAR sensor on a vehicle and detecting feature points on the ground by sensing the surroundings, thereby determining the absolute position precisely.

LiDAR sensors are used in combination with millimeter-wave radar and image sensors in the automatic operation of automobiles, and although they are unlikely to be used in the same manner in the case of railways, this is nevertheless important in grasping the performance of the single LiDAR sensor.

We therefore installed a LiDAR sensor on a train to study its detection of humans and cars. Through validation of its independent performance in addition to its potential for absolute position detection, we tried to assess its effectiveness as an obstacle detection device for the future. The probability of accidents is higher in the operation of trams when a car is approaching to turn right onto a tram track at an intersection. In addition, accident situation often causes tram delay. For these reasons, the basic experiments were carried out in order to ascertain the possibility of using the LiDAR sensor to judge the approach of such a car. The results are reported here.

\section{THE LIDAR SENSOR}

\subsection{Three-dimensional space identification sensor}

In recent times, the automobile industry has developed various sensors for automatic driving. Image sensors, millimeter-wave radar and LiDAR sensors are some types of three-dimensional space recognition sensors.

Among these, the LiDAR sensor, as opposed to the image sensor, is capable of recognition as long as it is within the range of the reflected light, whether in complete darkness or in a bright environment, where image sensors may become saturated. Further, the millimeterwave radar cannot achieve a resolution comparable to that of LiDAR as the wavelength of radio waves is longer than that of light. On the other hand, the current LiDAR sensor contains moving parts such as a motor for scanning the beam, which is deterrent when it comes to cost reduction or size reduction. However, with the development of mechanical LiDAR sensors that do not use a motor, the cost and size are both expected to decrease in the future.

\subsection{LiDAR sensor used in experiment}

The measurement principle of the LiDAR sensor is shown in Fig. 1, and Table 1 shows the specifications of the LiDAR sensors of two companies. These sensors were mounted on board and used for the experiments. Since both companies have two-dimensional as well as three-dimensional sensors, we confirmed four types of sensors in all, as shown in Table 1 and Fig. 2.

\section{DESCRIPTION OF EXPERIMENT}

\subsection{Experimental process}

- Test date: 15 September 2017, 11 a.m.-4 p.m.

- Location: Toyohashi Railway Co., Ltd., City Line

- Operation: two round trips between Akaiwaguchi and Ekimae stops two round trips between Azumadasakaue and Ekimae stops 


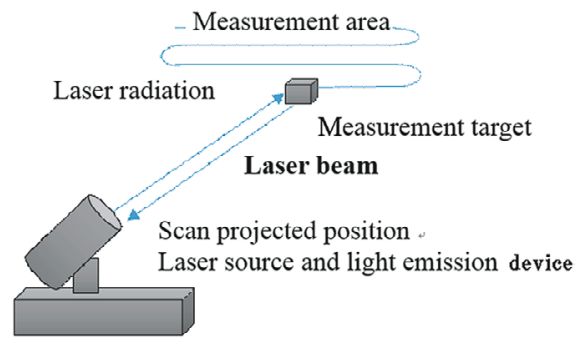

Figure 1: Measurement principle of LiDAR sensor [4].

Table 1: Specifications of LiDAR sensors used.

\begin{tabular}{|c|c|c|c|c|}
\hline \multirow[t]{3}{*}{ Type } & \multicolumn{2}{|c|}{ SICK models } & \multicolumn{2}{|c|}{ HOKUYO models } \\
\hline & $2 \mathrm{D}$ & $3 \mathrm{D}$ & $2 \mathrm{D}$ & $3 \mathrm{D}$ \\
\hline & $\begin{array}{l}\text { LMS511 } \\
{[5]}\end{array}$ & $\begin{array}{l}\text { LD-MRS } \\
{[6]}\end{array}$ & $\begin{array}{l}\text { UXM-30LHX- } \\
\text { EWA [7] }\end{array}$ & $\begin{array}{l}\text { YVT-35LX } \\
{[8]}\end{array}$ \\
\hline Laser wavelength (nm) & 905 & 905 & 905 & 905 \\
\hline Laser class & Class 1 & Class 1 & Class 1 & Class 1 \\
\hline Horizontal scan angle degrees & 190 & $85(110)$ & 190 & 210 \\
\hline Vertical scan angle degrees & - & 3.2 & - & 40 \\
\hline Horizontal scan rate $\mathrm{Hz}$ & $25-100$ & $12.5-50$ & 20 & 20 \\
\hline Vertical scan rate $\mathrm{Hz}$ & - & $\begin{array}{l}\text { Simultaneous } \\
\text { scanning }\end{array}$ & - & 1,200 \\
\hline Angular resolution (degrees) & $1 / 6$ variable & $1 / 8$ variable & 0.125 & Note 1 \\
\hline $\begin{array}{l}\text { Detection distance }(\mathrm{m}) \\
(10 \% \text { reflectance })\end{array}$ & 40 & 50 & 30 & 11 \\
\hline
\end{tabular}

Note 1: At least 2,590 points (20 FPS without interlace).

\subsection{Items of experiment}

The wayside landscape and the following test items were checked with the LiDAR sensor installed on the trams.

1. Detection of absolute position

(1) Side wall of each stop (Fig. 3)

(2) Wayside landscape

2. Validation as an effective obstacle detection device

(1) Tram (Fig. 4)

(2) Humans at the tram stops: three stops (Toyohashi Koenmae, Shiyakushomae, Ekimae Odori stops) (Fig. 5)

(3) Checking for a car turning right at an intersection 


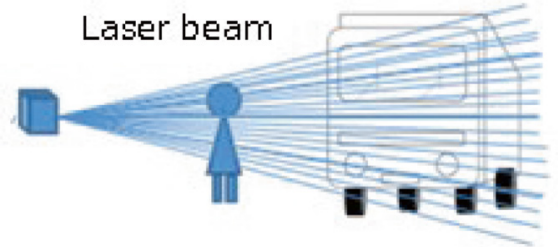

(a) HOKUYO 3D LiDAR sensor

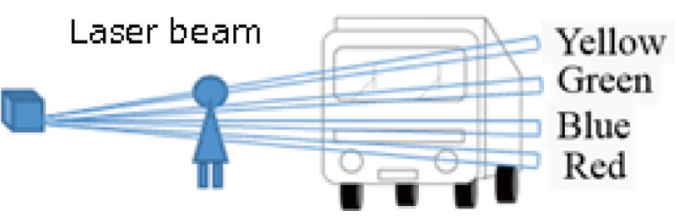

(b) SICK 3D LiDAR sensor

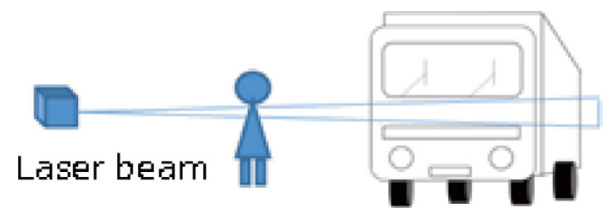

(c) 2D LiDAR sensor of both companies

Figure 2: Method of each sensor.

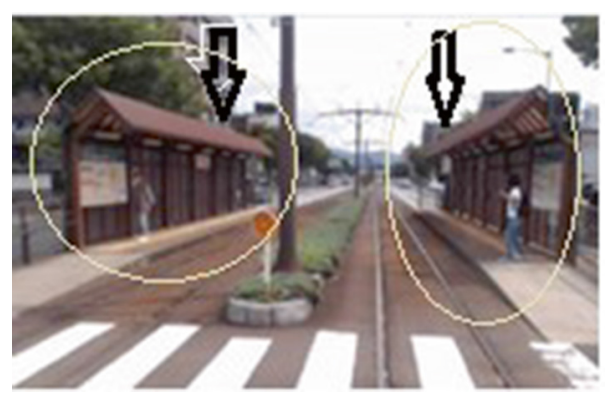

Figure 3: Sidewall of train stop.

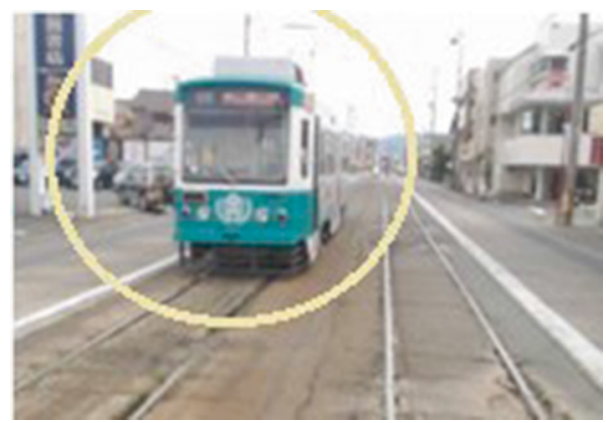

Figure 4: Tram. 


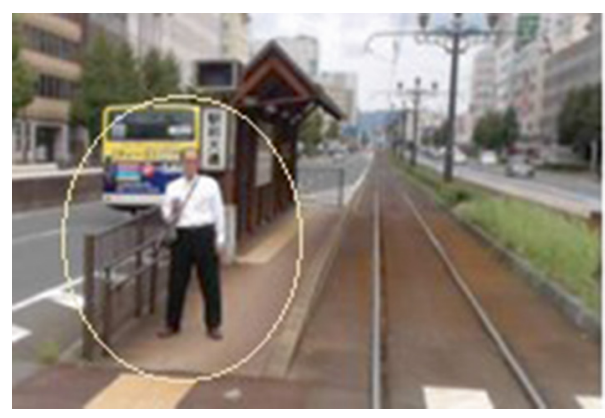

Figure 5: Humans at tram stop.

\subsection{Placement of LiDAR sensor}

Since the LiDAR sensor can penetrate even glass to make measurements, it was installed in the driver's cabin, as shown in Figs 6 and 7. SICK LiDAR sensors of the two- and threedimensional types were installed in the driver's cabin of the Akaiwaguchi bound, while twoand three-dimensional HOKUYO sensors were installed in the cabin of the Ekimae bound .

\section{MEASUREMENT RESULTS}

\subsection{Output results of LiDAR sensors}

The output results of the two-dimensional LiDAR sensor with regard to the side wall of the tram stop are shown in Figs. 8 and 9. Figures 10 and 11 show the output results of the threedimensional sensor.

As can be seen from Figs 8 and 9, the two-dimensional sensors of both companies recognized the side wall of the tram stop. While part of the laser beam is reflected by the glass, part of it permeates the glass and is reflected by the target ahead. The green color in Fig. 8 and red color in Fig. 9 indicate the results of reflection subsequent to the reflected light from the glass.
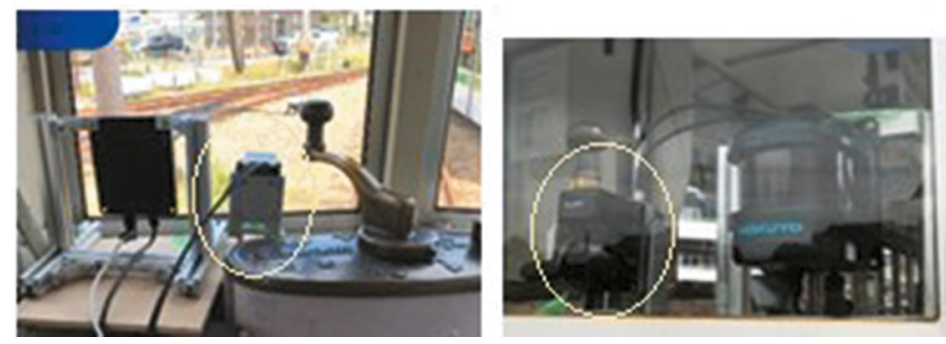

Figure 6: HOKUYO LiDAR sensor. 


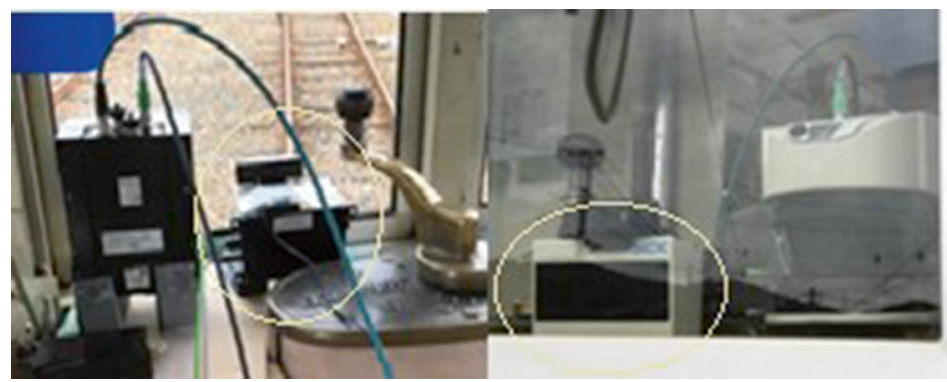

Figure 7: SICK LiDAR sensor.

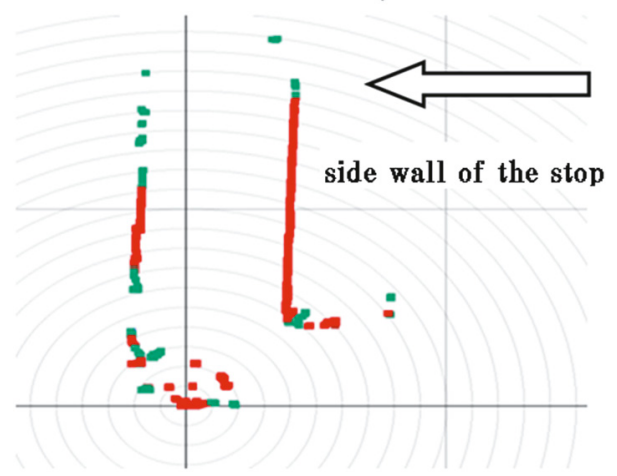

Figure 8: HOKUYO 2D sensor.

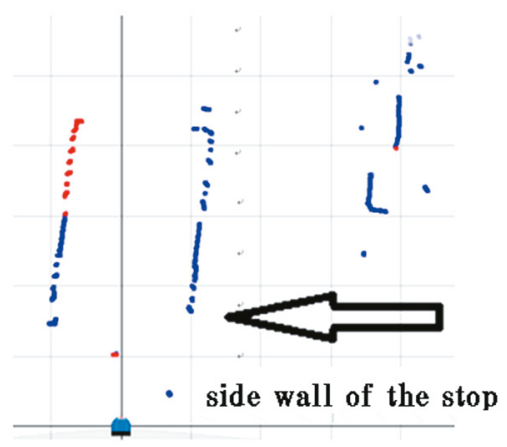

Figure 9: SICK 2D sensor.

For the three-dimensional sensors, the targets were different for the HOKUYO and SICK types. As shown in Fig. 10, the HOKUYO sensors were characterized by shape recognition at a short distance, and were capable of detecting the forms of humans and the fence of the tram stop. It was therefore concluded that the HOKUYO sensor is suitable for the detection of humans in the vicinity of a vehicle stop. 


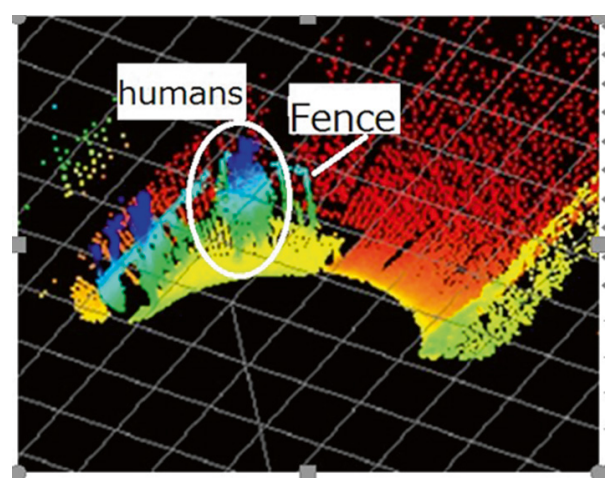

Figure 10: HOKUYO 3D sensor.

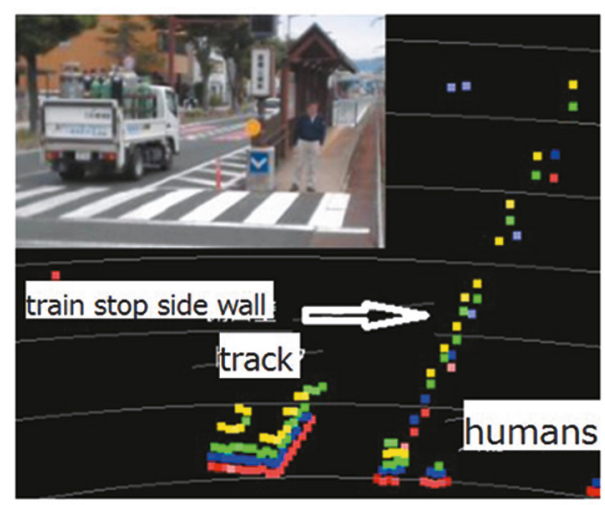

Figure 11: SICK 3D sensor.

On the other hand, in the case of the SICK sensors, four vertical layers appeared, as shown in Fig. 2b. The results are color-coded according to height to indicate the three-dimensional form. The test results are shown in Fig. 11. The characteristic feature of this sensor is its ability to measure long distances rather than the recognition of fine shapes.

\subsection{Absolute detection of wayside landscape}

Based on the above results, measurements of the wayside scenery were made using SICK's three-dimensional LiDAR sensor and HOKUYO's two-dimensional LiDAR sensor. The SICK three-dimensional LiDAR sensor measured the red building on the left at the turn at Azumadasakaue, as shown in Fig. 12. Six measurements were made during the passage of each operation, and the recognition results were extracted. As seen in Fig. 12, the red building on the left of the photograph was captured in the same manner all six times. These six measurement results were obtained at different speeds, and the maximum speed is set at $30 \mathrm{~km} / \mathrm{h}$. These results also showed that the LiDAR sensor was able to measure the red building regardless of train speed. 

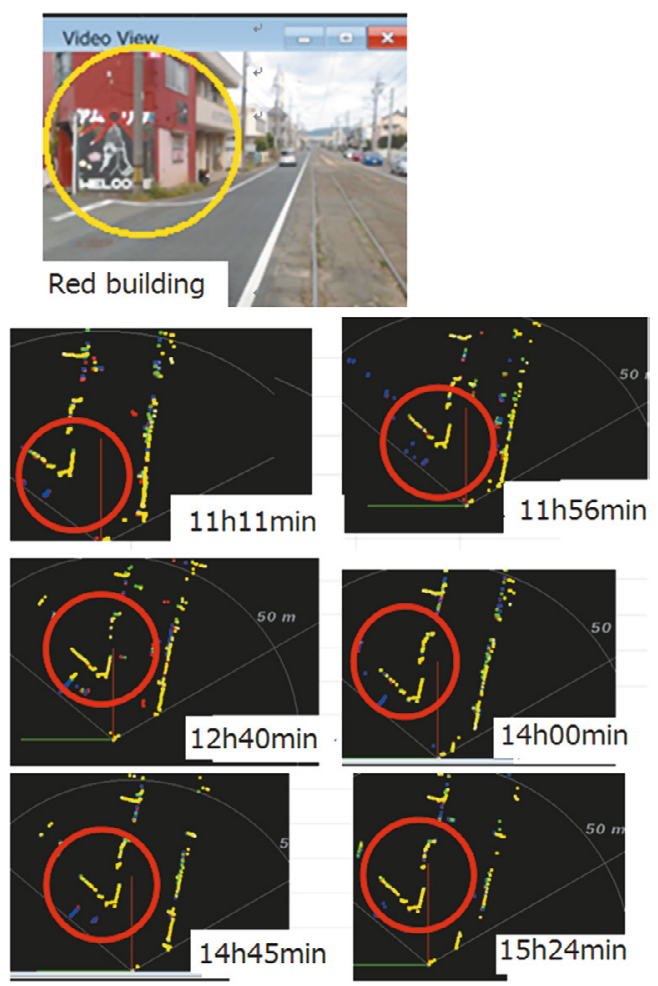

Figure 12: Measurement result of red building by 3D LiDAR sensor.

Figure 13 shows the results of measurement of the HOKUYO two-dimensional LiDAR sensor. These are the results of the recognition of buildings between Maehata stop and Higashihaccho stop. In this case too, the results of all four measurements are identical.

These results reveal the possibility of detecting the absolute position using a LiDAR sensor.

\subsection{Validation as an obstacle detection device}

Next, we confirmed the effectiveness of the sensor as an obstacle detection device.

1. Detection of tram

Since the front of the tram is large, a good reflection from its surface makes the extraction of features easy. Hence, the facing tram was set as the target object. The test result of the maximum distance recognition showed that despite measuring through glass, the recognition was up to a distance of 50-60 $\mathrm{m}$ in the case of the two-dimensional LiDAR sensor and 100-120 $\mathrm{m}$ in the case of the three-dimensional SICK LiDAR sensor.

2. Detection of human presence at tram stop

Testing for human presence was performed at three stops (Toyohashi Koenmae, Shiyakushomae and Ekimae Odori stops), but as the people were standing near a sign post, it was difficult to distinguish them from the post. However, the three-dimensional HOKUYO sensor was found to be capable of obtaining detailed shape information such as human forms within a radius of about $6 \mathrm{~m}$, as seen in Fig. 10. 


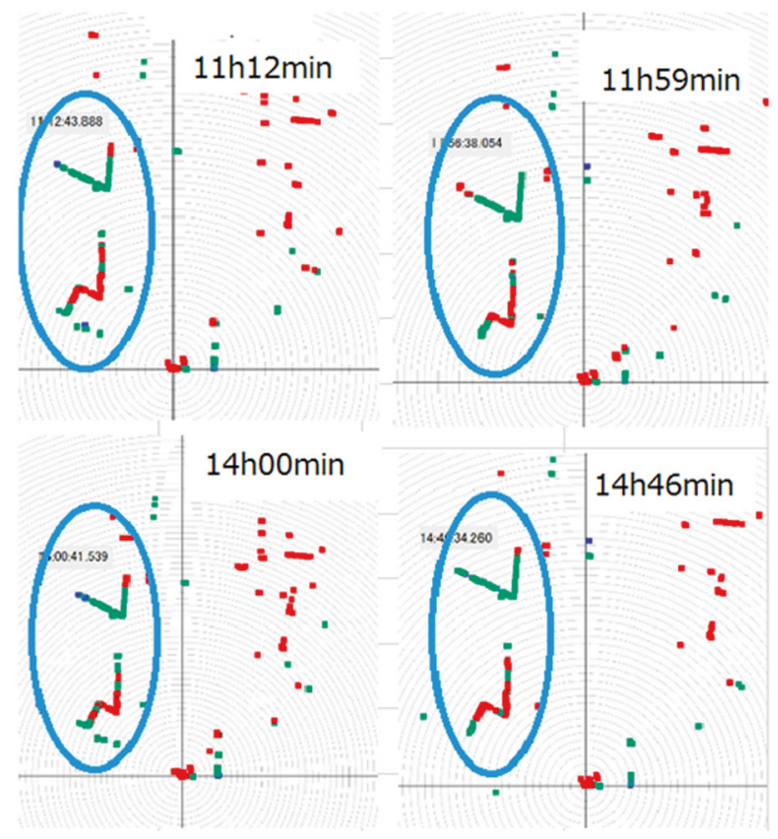

Figure 13: Result of HOKUYO 2D LiDAR sensor.

\section{Automobiles turning right}

The area that needs maximum caution during the operation of a tram is when it passes through an intersection, where cars taking a right turn are likely to enter onto the track. Therefore, the situation of a car waiting to make a right turn at an intersection and the scene of its entering onto the track was detected.

Figure 14 shows the situation of a bus waiting to make a right turn at an intersection, and the measurement result of the SICK three-dimensional LiDAR sensor. Figure 15 shows the result of the recognition of the cars at the intersection by the HOKUYO two-dimensional LiDAR sensor and the SICK three-dimensional LiDAR sensor. Both sensors were able to detect the form of the cars. We believe that these measurement results may be utilized to judge the entry of a car onto a track by the use of LiDAR sensors.
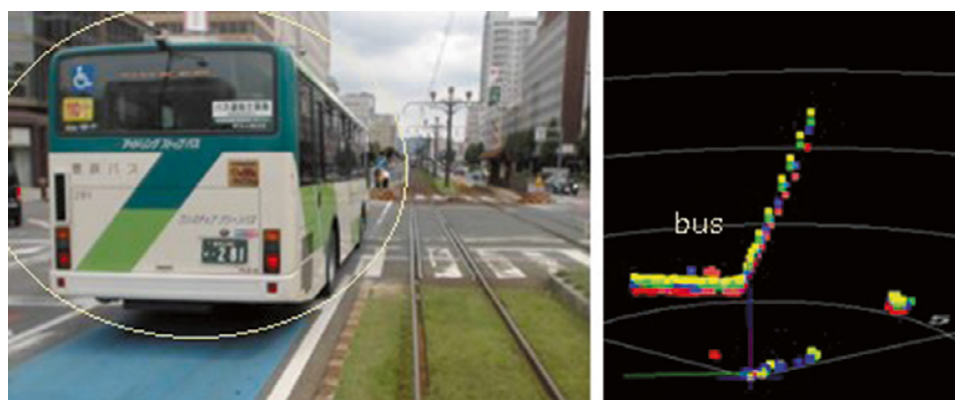

Figure 14: Bus making a right turn. 


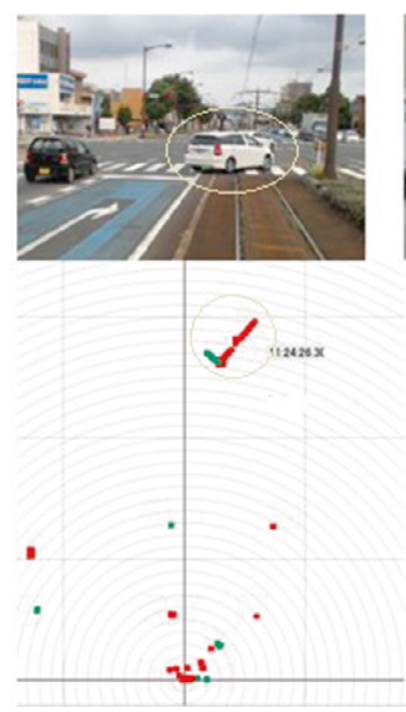

(a) HOKUYO 2D sensor

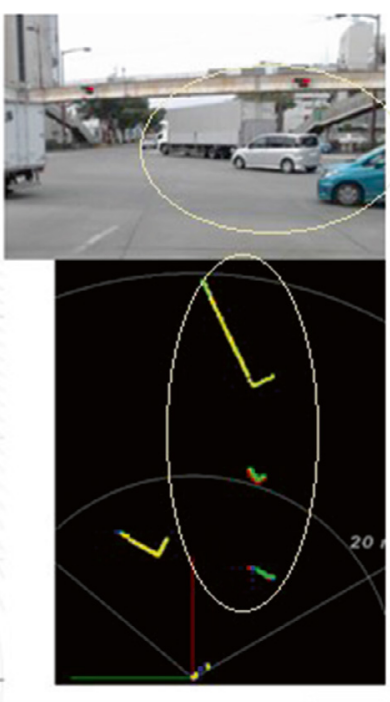

(b) SICK 3D sensor

Figure 15: Car entering an intersection.

\section{CONCLUSION}

As a measure to reduce the amount of equipment in railways, the onboard detection of train positions using satellite positioning was examined. As a method to detect the absolute position, a LiDAR sensor was mounted on board, and the possibilities of absolute position detection and the detection of obstacles on the track were investigated.

We first found that the absolute position of the vehicle could be determined by sensing the sidewalls of the tram stops or some specific objects in the wayside landscape.

Next, with regard to its utilization as an obstacle detecting device, we learned that it is possible to check whether an automobile has entered onto the track. As a future endeavor, we would like to construct a system that will calculate the distance of an object from the center of the track and raise an alarm when there is a risk of it coming into contact with the train.

\section{ACKNOWLEDGEMENTS}

We would like to thank Toyohashi Railway Co., Ltd., who cooperated with us by operating test vehicles for this experiment, and Hokuyo Automatic Co., Ltd., and SICK Co., Ltd., for providing the sensors.

\section{REFERENCES}

[1] Shinoda, N. \& Kudo, N., Study on processing method when using satellite positioning for train control. Joint Symposium on Railway Technology (J-Rail 2017), S7-5-1, 2017 (in Japanese), Niigata, Japan.

[2] Shinoda, N. \& Kudo, N., Study complement method when using a satellite positioning to train control. Joint Symposium on Railway Technology (J-Rail 2016), S2-10-5, 2016 (in Japanese), Tokyo, Japan. 
[3] Kojima, Y., Takahashi, A., Ninomiya, Y., Precise ego-localization estimation method by integration using standard GPS and an onboard scanning laser radar. IPSJ Journal, 50(1), pp. 64-74, 2009 (in Japanese). IPSJ-JNL5001007.pdf.

[4] Matsumoto, N., LiDAR, Price Destruction, Nikkei Electronics: Ridderkerk, pp. 49-55, 2017.

[5] SICK Co., Ltd., available at https://www.sick.com/media/pdf/1/41/941/dataSheet_ LMS511-1010000100_PRO_1046135_en.pdf, 2012 (accessed 1 September 2017).

[6] SICK Co., Ltd., available at https://www.sick.com/media/pdf/2/42/942/dataSheet_LDMRS400001S01_1052960_en.pdf, 2010 (accessed 1 September 2017).

[7] Hokuyo Automatic Co., Ltd., available at https://www.hokuyo-aut.co.jp/dl/Catalog_01_UXM-30LXH-EWA_1498439370.pdf, 2016 (accessed 1 September 2017).

[8] Hokuyo Automatic Co., Ltd., available at https://www.hokuyo-aut.co.jp/dl/Catalog YVT-35LX_MRS-0102A.pdf, 2017 (accessed 1 September 2017). 